\title{
Quality versus accuracy: result of a reanalysis of protein-binding microarrays from the DREAM5 challenge by using BayesPI2 including dinucleotide interdependence
}

\author{
Junbai Wang
}

\begin{abstract}
Background: Computational modeling transcription factor (TF) sequence specificity is an important research topic in regulatory genomics. A systematic comparison of 26 algorithms to learn TF-DNA binding specificity in in vitro protein-binding microarray (PBM) data was published recently, but the quality of those examined PBMs was not evaluated completely.

Results: Here, new quality-control parameters such as principal component analysis (PCA) ellipse is proposed to assess the data quality for either single or paired PBMs. Additionally, a biophysical model of TF-DNA interactions including adjacent dinucleotide interdependence was implemented in a new program - BayesPI2, where sparse Bayesian learning and relevance vector machine are used to predict unknown model parameters. Then, 66 mouse TFs from the DREAM5 challenge were classified into two groups (i.e. good vs. bad) based on the paired PBM quality-control parameters. Subsequently, computational methods to model TF sequence specificity were evaluated between the two groups.

Conclusion: Results indicate that both the algorithm performance and the predicted TF-binding energy-level of a motif are significantly influenced by PBM data quality, where poor PBM data quality is linked to specific protein domains (e.g. $\mathrm{C}_{2} \mathrm{H}_{2}$ DNA-binding domain). Especially, the new dinucleotide energy-dependent model (BayesPI2) offers great improvement in testing prediction accuracy over the simple energy-independent model, for at least $21 \%$ of analyzed the TFs.
\end{abstract}

\section{Background}

Recently, a comprehensive evaluation of 26 algorithms, for modeling transcription factor (TF) sequence specificity in in vitro protein-binding microarray (PBM) data [1], was published by DREAM5 (the Dialogue for Reverse Engineering Assessments and Methods) consortium. Many interesting results were revealed through this work. For example, mononucleotide position weight matrices (PWM) methods perform similarly to more advanced dinucleotide PWM algorithms for modeling TF sequence specificity, and inferred binding energy-level of a motif has little effect on overall prediction accuracy. This study also briefly mentioned that PBM data quality may have a strong influence on algorithm performance

Correspondence: junbai.wang@rr-research.no

Pathology Department, Oslo University Hospital - Norwegian Radium Hospital, Montebello 0310 Oslo, Norway across 66 mouse TFs. However, the actual data quality of the examined PBMs in the DREAM5 challenge (i.e. 66 training PBMs and 66 testing PBMs for the mouse TFs) is not investigated systematically. Generally, the microarray experiment is known for containing many kinds of biases $[2,3]$ such as nonlinearity, saturation, and dynamic range problems for the signal intensity. In DREAM5 challenge, for a pair of training and testing PBM experiments, two different array designs were used for a mouse TF. However, 8-mers that were used to compute the 8-mer median intensities for every PBM are identical. This unique feature provides an opportunity to assess the PBM data quality [4]. For instance, if both training and testing PBM experiments in good data quality, then the observed 8-mer median intensities between the training and testing PBMs will have good agreement. On the contrary, if one of the PBMs yields poor data 
quality, then the 8-mer median intensities between two PBMs will not match well. Consequently, the testing prediction accuracy is not a true reflection of the algorithm performance if paired PBMs have poor measurement agreements. In other words, computational algorithms will not predict a binding signal that only exists in the testing PBM experiment but it does not appear in the training PBM data, and vice versa. Thus, it is important to develop PBM quality-control parameters that can evaluate the data quality for either single or paired PBMs.

Free-energy-based biophysical modeling TF sequence specificity, from detailed theoretical studies [5-7] to rapid computational development in real applications [8-11], have been investigated for many years and several computer programs are publically available now [11-14]. Recently, dependent energy correction such as dinucleotide interdependence was also incorporated into TF-binding energy by BEEML-PBM and FeatureREDUCE [1]. In the DREAM5 challenge, performance of the dinucleotidedependent model of the two new programs is not improved greatly over the simple energy-independent model (i.e. $<10 \%$ of examined TFs were benefited by the energydependent model; increase in correlation coefficient $>0.05$ [1]). However, in many earlier studies, sequence dependencies in TF-binding sites were widely observed [15-18]. Particularly, energy-dependent model needs to fit a large number of unknown model parameters, which often encounters the over-fitting data problem that impairs the algorithm performance [19]. Additionally, if the input data is large, then there is a memory issue to $R$ and MATLAB programs which suffer from extremely slow computation (i.e. BEEML-PBM and many other programs in the DREAM5 challenge [1]). Therefore, it is worthy to design a novel algorithm which implements the dependent energy correction in an efficient programming language. Then, PBMs of 66 mouse TFs from the DREAM5 challenge can be reanalyzed by the new program. It may help revealing whether the limitation of previous algorithms hampers the discovery of motifs that contain nucleotide dependency in the binding sites.

Motivated by the above-mentioned challenges, new quality-control parameters for both single and paired PBMs, and a novel C implementation of biophysical modeling protein-DNA interactions including dinucleotide interdependence (BayesPI2) are presented here. The new methods and programs were applied on 66 mouse TFs in in vitro PBM experiments from the DREAM5 challenge. Overall, four major questions are investigated in this work: 1) the true data quality of paired PBM experiments for 66 mouse TFs; 2) the association between the PBM data quality and the algorithm performance; 3 ) whether the binding energy-dependent model offers a great improvement over the simple energy-independent model in testing prediction accuracy; 4) whether the low binding energy-level of a motif is a real biological phenomenon or a bias due to the data quality and the algorithm limitation.

\section{Results}

\section{PBM data quality of two mouse TFs}

The new PBM quality-control parameters were first tested at TF_7 (Mix) and TF_63 (Zkscan5), since they are extreme cases in the previous algorithm performance comparison [1]. For example, in Figure two of the original publication, 66 mouse TFs were sorted in decreasing order by the mean final algorithm performance scores, TF_7 and TF_63 were ranked as the first and the last TF, respectively. It suggests that the majority of evaluated algorithms performed significantly better at TF_7 than at TF_63 in the original study. In other words, the PBM data quality of TF_7 may be much better than that of TF_63. Thus, a quality analysis of the above-mentioned two TFs may tell the usefulness of new PBM quality-control parameters.

Figures $1 \mathrm{~A}$ and $1 \mathrm{~B}$ show the MA plots [2] of single PBM quality (training experiment) for TF_7 (Mix) and TF_63 (Zkscan5), respectively. In the MA plots, the higher the algorithm performance rank order of a TF, the longer the length of the major axis of the PCA ellipse (i.e. $\sim 5.3$ and $\sim 3.6$ for $\mathrm{TF}_{-} 7$ and TF_63, respectively). The major axis of the PCA ellipse is related to the orthogonal regression line between the background signal and PBM binding signal, which indicates the dynamical range of measured PBM signal intensities. If a PBM experiment has larger dynamical range, then better separation between the noise background signal and the true binding signal is achieved. Consequently, a higher TF rank order will be obtained in algorithm performance evaluation. The present results support assumption that the longer the length of the major axis the better the PBM data quality.

Figure $1 \mathrm{C}$ and $1 \mathrm{D}$ illustrate the quality of paired PBMs for TF_7 and TF_63, by applying PCA ellipse on the scatter plot of Z-score transformed and log normalized 8-mer median intensities between the two PBMs. In the scatter plots, the lengths of both the major and minor axes of the PCA ellipse are quite different between TF_7 (i.e. 9.4 and 2.4; Figure 1C) and TF_63 (i.e. 8.3 and 5.0; Figure 1D). This is consistent with the previous hypothesis in single PBM experiment that the lengths of the major and minor axes reflect the dynamical range of PBM signal intensities, and the difference of 8-mer median intensities between paired PBMs, respectively. Put differently, if a paired PBMs has good data quality (i.e. TF_7), then a PCA ellipse with long major axis but short minor axis will be expected. For paired PBMs, correlation coefficients of normalized 8-mer median intensities are also quite different between the good-quality PBMs (i.e. 0.87; TF_7) and the bad-quality ones (i.e. 0.46; TF_63). Nevertheless, it is not an indicator of measurement agreement 

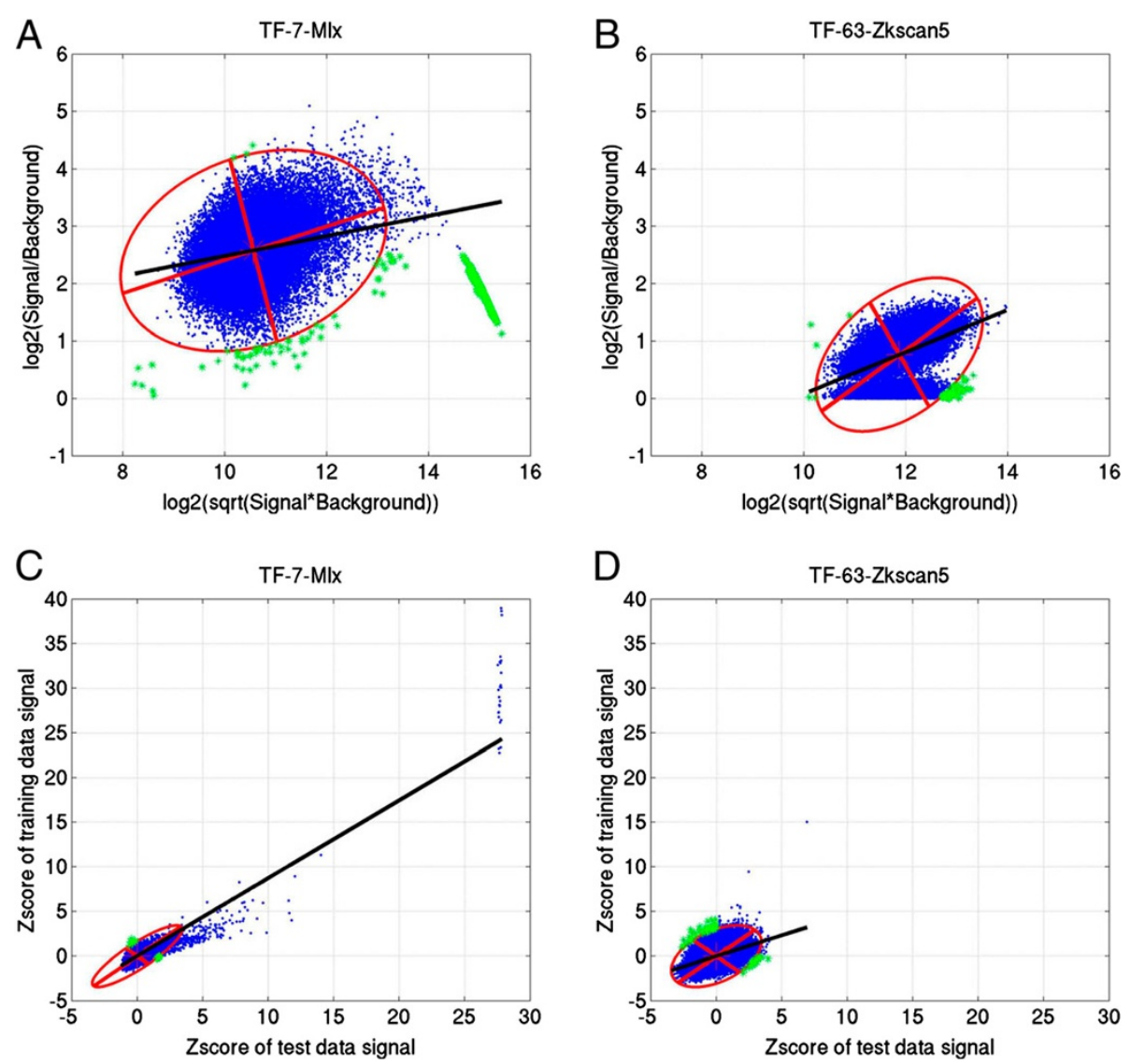

Figure 1 PBM data quality for TF_7 (Mix) and TF_63 (Zkscan5). A and B are MA scatter plots of TF_7 and TF_63, respectively. C and D are scatter plots of Z-score transformed 8-mer median intensities between a pair of training and testing PBM experiments for TF_7 and TF_63, respectively. In the figure, the red ellipses are $99.73 \%$ limit of PCA quality-control ellipses, the two red smooth lines are the length of the major and minor axes of the PCA ellipses, the black smooth lines are fitted linear regression lines, and the green data points are observations that may be out of the quality control (i.e. data point outside of the PCA ellipse and one of the observations is below sample mean).

between the two PBMs, because correlation coefficients measure the strength of a relationship between the two variables, and data with obvious poor agreement can produce high correlations [20]. Therefore, the new PBM quality-control parameters not only provided a visual inspection of data quality for either single or paired PBMs, but also suggested that TF rank order of algorithm performance comparison [1] is associated with the data quality of both training and testing PBMs.

\section{PBM data quality of 66 mouse TFs}

Encouraged by the above observations, it is necessary to investigate all 66 TFs that have paired PBMs. First, scatter plots of TF rank order vs. the single PBM (training data) data quality are shown in Figure 2, where $x$-axis is the sorted rank order of 66 TFs based on the algorithm performance comparison in Figure 2 of original paper [1], and $y$-axis is the single PBM quality parameter for 66 training $\mathrm{PBM}$ experiments such as the length of the major and minor axes of the PCA ellipse (Figure 2A and 2B), correlation coefficient (Figure 2C), and regression coefficient (Figure 2D). A linear regression line was fitted to every scatter plot, where P-values to regression coefficients for the length of the major axis of the PCA ellipse, the correlation coefficient, and the regression coefficient are $\mathrm{P}<$ $0.00014, \mathrm{P}<0.00021$, and $\mathrm{P}<0.063$, respectively. The results suggest that the quality of training PBM experiments is significantly correlated to the TF rank order of mean algorithm performance comparison. Nevertheless, in a similar study by 66 testing PBM experiments, most of the single PBM quality parameters are not linked to the TF rank order Additional file 1: Figure S1, except for the correlation coefficient between the signal intensities and the background intensities $(\mathrm{P}<0.0022)$. It indicates that the algorithms may have been learning some background signals, since in general they performed best on training or testing sets where the signal and background intensities are highly correlated. For that reason, algorithm performance comparison of the original paper [1] was swayed by the quality of PBM training data (i.e. Figure $2 \mathrm{~A}$ and $2 \mathrm{C}$ ). 

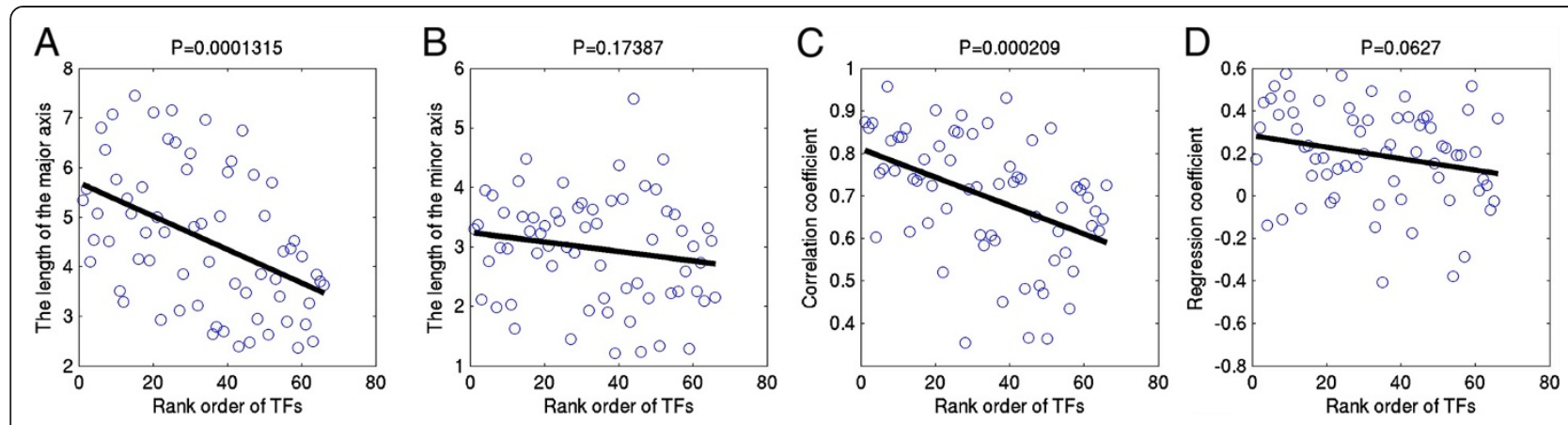

Figure 2 Scatter plots of algorithm performance rank order versus PBM training data quality. A, B, C, and D show scatter plots of algorithm performance rank order of 66 mouse TFs versus the length of the major axis of the PCA ellipse (i.e. 99.73\% limit of PCA quality control ellipse), the length of the minor axis of the PCA ellipse, correlation coefficient between signal intensities and background intensities, and regression coefficients, respectively. Both PCA ellipse and regression coefficient are based on MA scatter plots for PBM training datasets. In the figure, the black smooth lines are fitted linear regression lines to the scatter plots, and the P-values of the regression lines are indicated at the top of each figure. The rank order of TFs (i.e. 66 TFs were sorted in decreasing order by mean final algorithm performance scores) is adopted from Figure 2 of the DREAM5 challenge paper.

Then, scatter plots of TF rank order versus the paired PBM quality parameters are illustrated in Figure 3, where a linear regression line was fitted to each plot. P-values to the regression coefficients are $\mathrm{P}<1.75 \times 10^{-13}, \mathrm{P}<1.96 \times$ $10^{-13}, \mathrm{P}<1.45 \times 10^{-13}$, and $\mathrm{P}<1.45 \times 10^{-13}$ for the length of the major axis (Figure 3A), and minor axis (Figure $3 \mathrm{~B}$ ) of the PCA ellipse, the correlation coefficients (Figure 3C), and the regression coefficients (Figure 3D) of normalized 8-mer median intensities between training and testing PBMs, respectively. The results are very interesting because all quality-control parameters of paired PBMs are significantly correlated to the TF rank order according to the mean algorithm performance comparison. It appears that the deterioration of mean algorithm performance across the 66 TFs (i.e. Figure 2 of the original publication [1]) is largely due to the decrease in agreement between the training and the testing PBMs. Specifically, computational methods for modeling TF sequence specificity are extremely sensitive to the data quality of both training and testing PBM experiments.

\section{Classifying 66 mouse TFs into two groups based on PBM data quality}

So far, the results of analyzing 66 mouse TFs imply that methods for modeling TF sequence specificity are strongly affected by the PBM data quality. It is better to group 66 TFs into two clusters (i.e. good versus bad quality) by PBM quality-control parameters, then to reevaluate the algorithm performance (i.e. BayesPI2 energy-independent model versus the energy-dependent model including dinucleotide dependence). As a consequence, 66 mouse TFs from the DREAM5 challenge were assigned to two clusters by applying unsupervised fuzzy neural gas methods on the single PBM quality parameters (i.e. training PBM experiment). Comparing to the known TF rank order from original work [1], the best classifications were achieved by two parameters
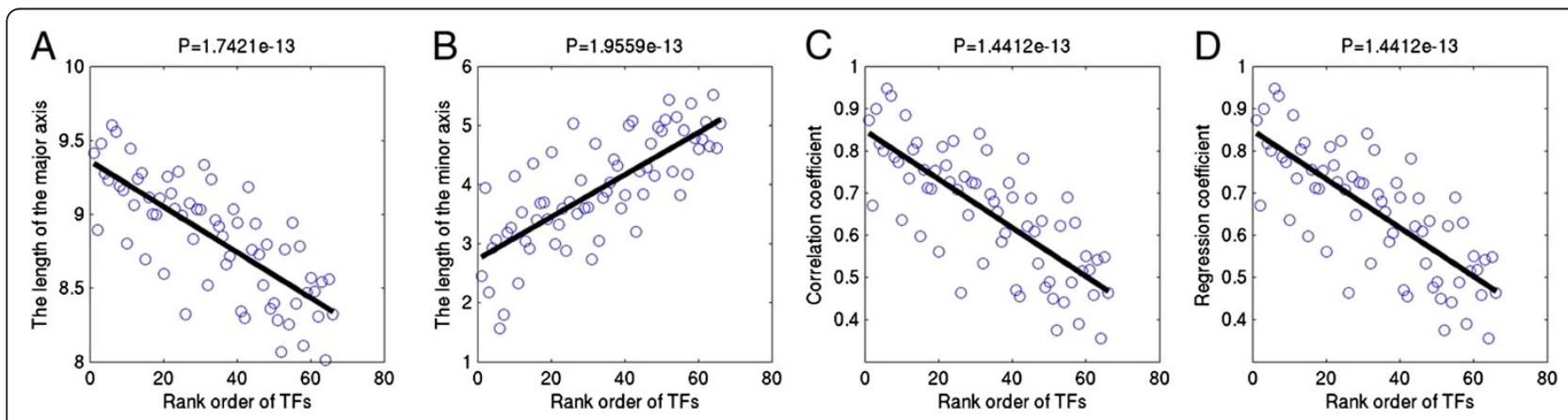

Figure 3 Scatter plots of algorithm performance rank order versus agreement of paired PBMs. A, B, C, and D show scatter plots of algorithm performance rank order versus the length of the major axis of the PCA ellipse (i.e. 99.73\% limit of PCA quality-control ellipse), the length of the minor axis of the PCA ellipse, correlation coefficient of normalized 8-mer median intensities between a pair of training and testing PBM experiments, and regression coefficients, respectively. Both PCA ellipse and regression coefficient are based on scatter plots of Z-score transformed 8-mer median intensities between a pair of training and testing PBM experiments. In the figure, the black smooth lines are fitted linear regression lines to the scatter plots, and the P-values of the regression lines are indicated at the top of each figure. The rank order of TFs (i.e. 66 TFs were sorted in decreasing order by mean final algorithm performance scores) is adopted from Figure 2 of the DREAM5 challenge paper. 
(i.e. correlation coefficient between normalized signal intensities and background intensities, and the length of the major PCA axis). That is consistent with the earlier observations in Figure $2 \mathrm{~A}$ and $2 \mathrm{C}$. The clustering result is an average of ten times classifications based on the above-mentioned two-quality parameters, where cluster one contains more good-quality PBM experiments than that in cluster two. For example, $\sim 79 \%$ of $24 \mathrm{TF}$ that grouped in the first cluster belong to the top half of ranked TFs (i.e. TF rank order from 1 to 33 ), and $\sim 67 \%$ of $42 \mathrm{TF}$ that assigned to the second cluster are in the bottom half of ranked TFs (i.e. TF rank order from 34 to 66). In summary, the quality of training PBM experiments influences the algorithm performance comparison. In other words, the algorithm performance on testing data may be predicted by the corresponding training data quality.

Then, the same 66 mouse TFs were classified into two clusters based on paired PBM quality parameters (i.e. agreement between training and testing PBMs). A combination of different quality parameters (i.e. the length of the major and minor axes of the PCA ellipse, regression coefficient, and correlation coefficients of normalized 8-mer median intensities between training and testing PBMs) were tested, and the best classification was obtained by using the lengths of both the major and minor axes of PCA ellipse, which characterize the dynamical range of $\mathrm{PBM}$ signal intensities and the difference of 8-mer median intensities between the two PBMs, respectively. The average of ten times classifications of 66 TFs by the two parameters is shown in Table 1 and Additional file 1: Table S1 for good and bad PBMs, respectively. In the tables, TFs were evenly assigned to two clusters: 34 TF were grouped in cluster one (Table 1) where $\sim 79 \%$ of them have algorithm performance rank order from 1 to 33; and 32 TF were grouped in cluster two (Additional file 1: Table S1) where $\sim 81 \%$ of them have algorithm performance rank order between 34 and 66 [1]. The results imply that the TFs of cluster one (Table 1) were mostly measured by paired PBMs with good data quality, but the TFs of cluster two (Additional file 1: Table S1) were frequently observed under poor-quality PBM experiments. Particularly, the classification based on the agreement of paired PBMs is much better than that done by single PBM data quality. It demonstrates that the quality of both training and testing PBMs plays a pivotal role in evaluating algorithm performance for computational methods to model TF sequence specificity. Thus, the new classification based on the agreement of paired PBMs will be utilized in future data analysis.

\section{Verification of BayesPI2 energy-dependent model including dinucleotide interaction energies}

In this study, biophysical modeling of protein-DNA interaction with dinucleotide interdependency is implemented in C - BayesPI2, by using techniques similar to sparse Bayesian learning and relevance vector machine. To test the new program, it was evaluated by two PBM datasets (Egr1 and Hnf4a), which are known to contain nucleotide interdependent effects on the binding affinities of TFs [17,21,22]. First, the protein-binding energy matrices (PBEMs) of both Egr1 and Hnf4a were estimated, by applying BayesPI2 on Z-score transformed and log-normalized probe intensities of one of the replicate PBMs (i.e. motif length ranges from 7 to 12). Then, the predicted PBEMs were used to estimate the TF-binding intensities on the other replicate PBMs. Binding energy matrices predicted by the BayesPI2 energy-independent model, which result in the highest correlation coefficient between the predicted intensities and the testing probe intensities, are shown in Figure 4A and $4 \mathrm{~B}$ for Egr1 (correlation coefficient 0.74) and Hnf4a (correlation coefficient 0.58), respectively. The best PBEMs calculated by BayesPI2 dinucleotide energydependent model are displayed in Figure 4C and 4D for Egr1 (correlation coefficient 0.77) and Hnf4a (correlation coefficient 0.71 ), respectively. The corresponding dinucleotide interaction energies are shown in the heatmaps, Figure 4E and 4F. It is clear that BayesPI2 energydependent model including dinucleotide interdependence improves testing prediction accuracy for both TFs. Especially, for Hnf4a, the improvement is striking (i.e. the difference of correlation coefficients between the energydependent and the energy-independent model is greater than 0.1), and the strongest dinucleotide interaction occurs at positions 6 and 7 (Figure 4D and 4F), which is consistent with a previous study [22] that applied BEEML-PBM on the same data. It is worthy to note that the dinucleotide interactions often appear at TFbinding sites with low binding energy (or information content), please refer to Figure $4 \mathrm{C}, \mathrm{D}, \mathrm{E}$, and F. Taken together, the new program - BayesPI2 by including dinucleotide energy-dependent model performs well towards the real PBM data.

\section{Applying BayesPI2 on 66 mouse TFs (good quality versus bad quality)}

Both BayesPI2 energy-independent model and dinucleotide energy-dependent model were applied on Z-score transformed and log-normalized probe intensities of 66 training PBMs [1]. The inferred TF-binding energy matrices were then used to evaluate prediction accuracies at 66 testing PBMs from the same DREAM5 challenge [1]. Results are shown in Table 1 and Additional file 1: Table S1 for TFs with good-quality PBMs and those with badquality PBMs, respectively. The two groups were classified earlier based on the paired PBMs quality parameters. For BayesPI2 energy-independent model, the median correlation coefficients between testing probe intensities and BayesPI2 predicted intensities are 0.67 and 0.45 in Table 1 
Table 1 Prediction results of TFs with good PBM quality by using BayesPI2 energy-independent model and energy-dependent model including dinucleotide interactions

\begin{tabular}{|c|c|c|c|c|c|c|c|c|}
\hline & TF family & Rank & CorrCoef (Ind) & Length (Ind) & Number (Ind) & CorrCoef (Dep) & Length (Dep) & Number (Dep) \\
\hline TF_7 & bHLH & 1 & 0.78 & 13 & 1 & 0.786 & 10 & 1 \\
\hline TF_26 & bHLH & 2 & 0.66 & 10 & 1 & 0.67 & 13 & 1 \\
\hline TF_56 & $\mathrm{C} 2 \mathrm{H} 2 \mathrm{Z}$ F(4) & 3 & 0.76 & 12 & 1 & 0.788 & 13 & 1 \\
\hline TF_55 & AT hook & 4 & 0.8 & 8 & 1 & 0.8 & 9 & 1 \\
\hline TF_17 & NR & 5 & 0.76 & 9 & 1 & 0.757 & 8 & 1 \\
\hline TF_11 & NR & 6 & 0.826 & 10 & 1 & 0.833 & 10 & 1 \\
\hline TF_16 & Myb/SANT & 7 & 0.808 & 10 & 1 & 0.817 & 9 & 1 \\
\hline TF_31 & $\mathrm{C} 2 \mathrm{H} 2 \mathrm{ZF}(13)$ & 8 & 0.585 & 12 & 1 & 0.59 & 11 & 1 \\
\hline TF_15 & Pou + Homeo & 9 & 0.62 & 11 & 1 & 0.655 & 11 & 1 \\
\hline TF_45 & Myb/SANT & 11 & 0.8 & 12 & 1 & 0.78 & 11 & 1 \\
\hline TF_42* & Forkhead & 12 & 0.75 & 12 & 1 & 0.805 & 13 & 1 \\
\hline TF_64 & $\mathrm{C} 2 \mathrm{H} 2 \mathrm{ZF}(3)$ & 13 & 0.75 & 8 & 1 & 0.75 & 10 & 1 \\
\hline TF_52 & $N R$ & 14 & 0.807 & 12 & 1 & 0.79 & 10 & 1 \\
\hline TF_3* & Forkhead & 16 & 0.67 & 10 & 1 & 0.724 & 11 & 1 \\
\hline TF_27* & bZIP & 17 & 0.526 & 9 & 1 & 0.635 & 11 & 1 \\
\hline TF_18 & Sox & 18 & 0.638 & 8 & 1 & 0.677 & 8 & 1 \\
\hline TF_22* & T-box & 19 & 0.675 & 9 & 1 & 0.746 & 12 & 1 \\
\hline TF_47 & Homeo & 21 & 0.726 & 12 & 1 & 0.767 & 11 & 1 \\
\hline TF_44 & GATA & 22 & 0.633 & 12 & 1 & 0.68 & 10 & 1 \\
\hline TF_28 & $\mathrm{C} 2 \mathrm{H} 2 \mathrm{ZF}(8)$ & 23 & 0.58 & 11 & 1 & 0.6 & 11 & 1 \\
\hline TF_13* & Pou + Homeo & 24 & 0.584 & 9 & 1 & 0.675 & 13 & 1 \\
\hline TF_5 & $\mathrm{C} 2 \mathrm{H} 2 \mathrm{ZF}(3)$ & 25 & 0.59 & 10 & 1 & 0.618 & 11 & 1 \\
\hline TF_43 & Forkhead & 27 & 0.577 & 10 & 1 & 0.614 & 12 & 1 \\
\hline TF_19 & Sox & 29 & 0.559 & 8 & 1 & 0.59 & 11 & 1 \\
\hline TF_39 & $\mathrm{C} 2 \mathrm{H} 2 \mathrm{ZF}(3)$ & 30 & 0.63 & 11 & 1 & 0.668 & 13 & 1 \\
\hline TF_51* & Pou + Homeo & 31 & 0.615 & 12 & 1 & 0.67 & 13 & 1 \\
\hline TF_23 & T-box & 33 & 0.64 & 12 & 1 & 0.66 & 13 & 1 \\
\hline TF_12* & NR & 35 & 0.55 & 13 & 1 & 0.606 & 13 & 1 \\
\hline TF_49 & $N R$ & 34 & 0.675 & 10 & 1 & 0.676 & 11 & 1 \\
\hline TF_53* & RFX & 39 & 0.696 & 12 & 1 & 0.77 & 13 & 1 \\
\hline TF_14 & Myb/SANT & 40 & 0.6 & 8 & 1 & 0.62 & 9 & 1 \\
\hline TF_48 & $N R$ & 43 & 0.755 & 12 & 1 & 0.78 & 12 & 1 \\
\hline TF_38 & DM & 45 & 0.67 & 9 & 1 & 0.689 & 8 & 1 \\
\hline TF_32 & $\mathrm{C} 2 \mathrm{H} 2 \mathrm{ZF}(6)$ & 55 & 0.587 & 9 & 1 & 0.62 & 8 & 1 \\
\hline
\end{tabular}

In the table, the 34 TFs were classified by applying fuzzy neuronal gas algorithm on the paired PBM quality-control parameters (i.e. the length of the major and minor axes of the PCA ellipses), where a good agreement between training and testing PBMs indicates good PBM data quality; Rank means TFs are sorted in decreasing order of their final performance score across all tested algorithms in Figure 2 of original publication [1]; CorrCoef , Length, and Number are Pearson correlation between predicted intensities and testing probe intensities, the length of motif, the first or second motif, respectively; (Ind) and (Dep) represent BayesPI2 energy-independent model and energy-dependent model including dinucleotide interaction, respectively; TFs marked by star and bold text indicate that the increase in Pearson correlation coefficient is greater than 0.05 by using BayesPI2 energy-dependent model including dinucleotide interaction energies.

and Additional file 1: Table S1, respectively. A two-tailed T-test of correlation coefficients between Table 1 and Additional file 1: Table S1 gives P-value $6.0958 \times 10^{-12}$, which suggests that algorithm testing prediction accuracy from Table 1 (i.e. 34 TFs with good agreement between training and testing PBMs) is significantly better than that from Additional file 1: Table S1 (i.e. 32 TFs with poor agreement between the two PBMs). Similar T-tests were carried out for algorithm performance scores (i.e. Pearson correlation coefficient of probe intensities) of 26 algorithms published by the DREAM5 challenge [1], more than $85 \%$ of algorithms show significant better performance at good 


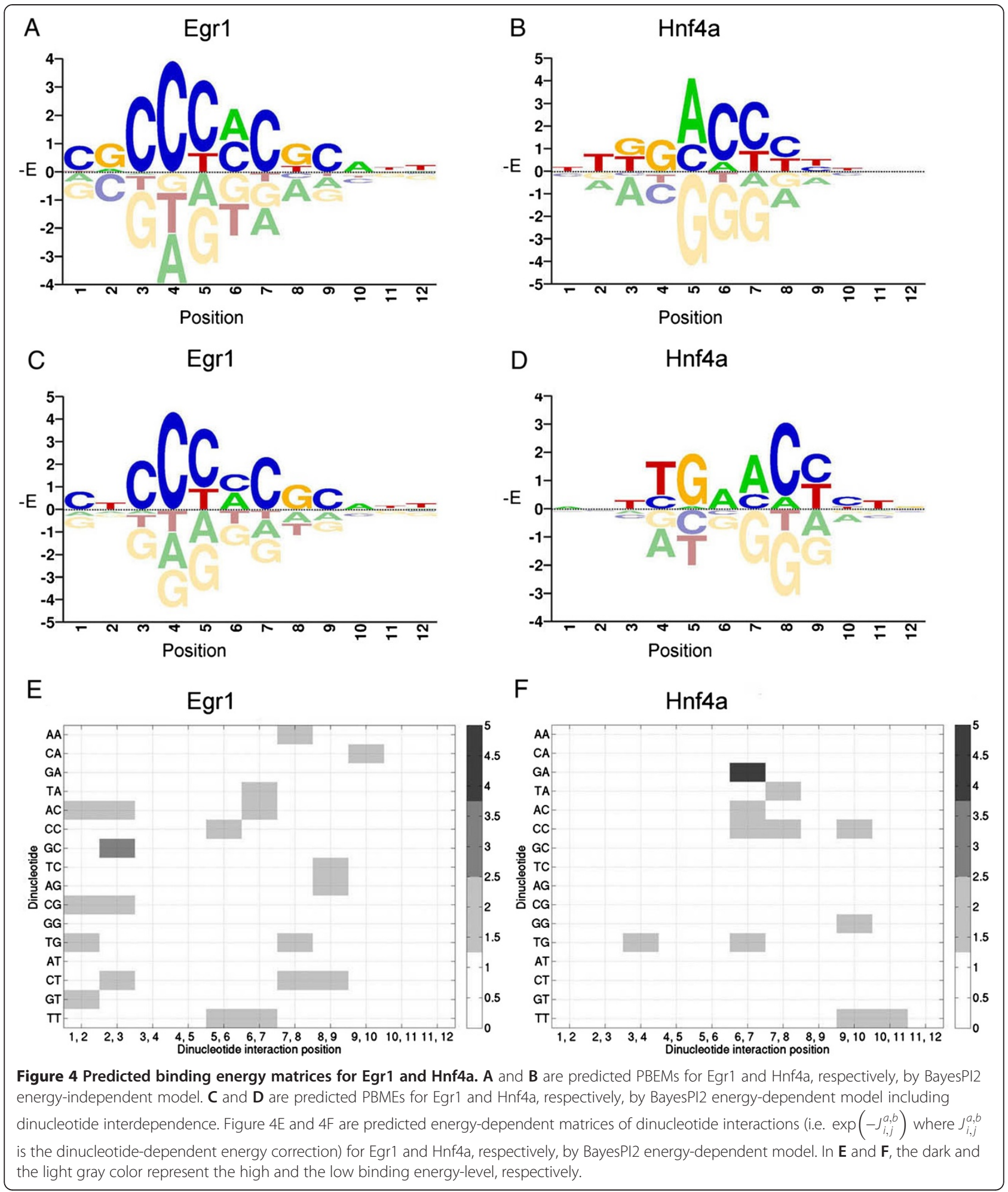

PBM quality group than that at poor PBM quality group (i.e. 22 and 23 algorithms with T-test P-value $<0.01$ in Additional file 1: Tables S2 and S3, respectively). Nevertheless, four algorithms seem to have little effect by the quality of PBMs, where k-mer sequence-specific model plus feature selections were used (i.e. Team_k, Team_B, Team_I, and Seed-and-Wobble).

Notable, in Table 1, 34 TFs are spread to almost 14 different DNA-binding domains such as bHLH (2 TFs) and $\mathrm{C}_{2} \mathrm{H}_{2}(7 \mathrm{Tfs})$. However, in Additional file 1: Table 
S1, 32 TFs just belong to 6 different DNA-binding domains where almost half of them (15 TFs) are $\mathrm{C}_{2} \mathrm{H}_{2}$ DNA-binding domain (Zinc finger protein). It indicates that the poor quality of some PBM experiments (i.e. Additional file 1: Table S1) may be protein domain specific (i.e. Zinc finger protein in Additional file 1: Table S1). Additionally, if we only consider protein domains with more than two TFs from both Table 1 and Additional file 1: Table S1, then there are three protein domains (i.e. around 67\%, 67\%, and 60\% of TFs from Forkhead, Pou + Homeo, and bZIP, respectively) that received a great boost in testing prediction accuracy (i.e. increase in correlation coefficients $>0.05$ ) after using BayesPI2 binding energy-dependent model. In other words, these three protein domains may more frequently encounter base pair interdependency in the DNA-binding sites than that in the other domains.

Results from both Table 1 and Additional file 1: Table S1 reveal that the testing prediction accuracy based on energy-dependent model is very sensitive to the data quality, and the Bayesian method is robust against the data noise. For example, by using the energy-dependent model of BayesPI2 or BEEML-PBM [1], about 14 and 5 TFs show great improvement (increase in Pearson correlation coefficient $>0.05$ ) in the testing prediction accuracy, respectively, over that by the simple energy-independent model; for the same test, no improvement was found by FeatureREDUCE. Among the 5 TFs provided by BEEML-PBM, 3 belong to the good-quality PBM group (i.e. TF_27, TF_32, and TF_53; Table 1) where 2 TFs (TF_27 and TF_53) were identified by BayesPI2, and the remaining 2 TFs are in the bad-quality PBM group (i.e. TF_21 and TF_60; Additional file 1: Table S1) where only one TF (TF_21) was recovered by BayesPI2. Thus, by applying various algorithms on the PBMs, the overlap of predictions is poor for TFs with badquality data but robust to TFs with good-quality ones. In summary, the better the PBM data quality, the better the testing prediction accuracy, and both the PBM data quality and the TF-binding site interdependency may be protein domain specific. Especially, the good-quality PBM experiments generally benefit more from biophysical modeling of protein-DNA interactions including dinucleotide interactions, than the poor ones from the same computation.

\section{Predicted TF-binding energy-level versus paired PBM data quality}

Encouraged by the above findings, it is interesting to investigate relationships between the predicted TF-binding energy-level of a motif and the PBM data quality across 66 TFs. First, for each TF, the median of negative binding energies of the first predicted binding energy matrix by BayesPI2 energy-independent model was computed. Then, a log-transformed absolute median energy value was used to summarize the binding energy-level of a motif, which is proportional to the information content of the motif. Scatter plots of the log-transformed median TF-binding energy-level against the sorted paired PBM quality parameters such as the length of the major and minor axes of the PCA ellipse, correlation coefficient and regression coefficient between training and testing PBMs, are displayed in Figure 5A, B, C, and D, respectively. A linear regression line was fitted to every scatter plot, P-value to the regression coefficient shows that the median binding energy-level of a motif is positively correlated to the length of the major axis of the PCA ellipse $(\mathrm{P}<0.003)$ and the correlation coefficients $(\mathrm{P}<0.0024)$, but anti-correlated to the length of the minor axis of the PCA ellipse $(P<0.0015)$. Results by applying BayesPI2 energy-independent and dinucleotide energy-dependent model (i.e. using either normalized 8-mer median intensities or probe intensities) are available in the (i.e. Additional file 1: Figures S2, S3, S4 and S5), where almost all of them show significant positive correlation between the median binding energy-level of a motif and the paired PBMs data quality, except for few cases (i.e. different motif length) obtained by applying BayesPI2 dinucleotide energy-dependent model on the PBM probe intensities. Hence, the better the PBM data quality, the higher the binding energy-level (or information content) of a motif.

To verify the present finding, the same analysis was performed again on a set of PWMs provided by the DREAM5 challenge [1], where the PWMs were predicted by various methods based on the same training PBM experiments for 66 mouse TFs. Methods that were used to obtain those PWMs include both biophysical free-energy models (i.e. BEEML-PBM [22], FeatureREDUCE, and MatrixREDUCE [11]) and other model types (i.e. PWM + HWMs from Team_E [1], and RankMotif [23]). First, each PWM was converted to TF-binding energy matrix. Then, scatter plots of log-transformed median binding energy-level of a PWM versus sorted paired PBM quality parameters were made (i.e. Figure 5E, F, G, and H). P-values of regression coefficients to the length of the major and minor axes of the PCA ellipse, and the correlation coefficients are $\mathrm{P}<0.03, \mathrm{P}<0.045$, and $\mathrm{P}<0.03$, respectively. It is clear that there is a strong positive correlation between the predicted binding energy-level of a motif and the PBM data quality. Overall, the results of published PWMs are consistent with the previous findings by using the BayesPI2 predicted binding energy matrices. It reinforces the hypothesis that methods for modeling TF sequence specificity are extremely sensitive to the PBM data quality. Specifically, the low energy-level (information content) of a predicted binding energy matrix may be caused by the poor PBM data quality (i.e. the poor agreement between training and testing PBMs). 


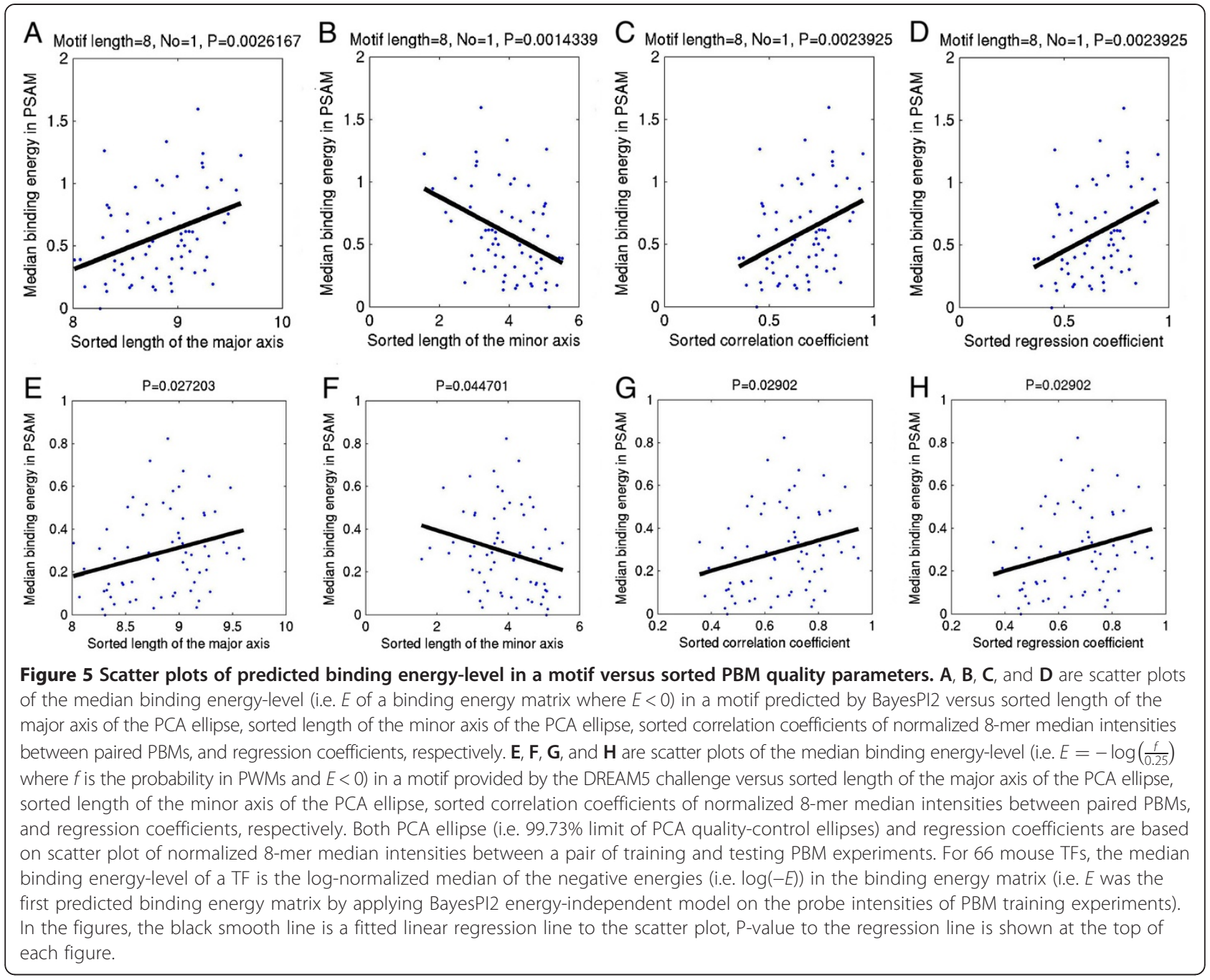

\section{Algorithm performance comparison and verification of predicted PBEM in ChIP-seq data}

A comparison of algorithm performance between the BayesPI2 and the other methods was carried out, where the median Pearson correlation coefficients (i.e. correlation between the predicted probe intensities and the actual intensities) of 66 mouse TFs were computed for each algorithm (Additional file 1: Table S4). The correlation coefficients based on bayesPI 2 and 26 other algorithms were obtained from this study (i.e. Table 1 and Additional file 1: Table S1) and the earlier publication [1] (i.e. Supplementary Table 3 of original paper), respectively. A scatter plot of the sorted median Pearson correlation coefficients for all algorithms is shown in Additional file 1: Figure S6 where the performance of BayesPI2 is close to the top 10 ranked algorithms from the original paper. However, it should be noted that the present comparison may not tell the true merit of each algorithm because of the poor data quality in PBM experiments (i.e. Additional file 2 versus supplementary
Figure 4 of original paper). For computational cost, BayesPI2 takes $\sim 7 \mathrm{~min}$ and $\sim 30 \mathrm{~min}$ to predict one PBEM (i.e. using $\sim 40000 \mathrm{PBM}$ probe sequences and $\sim 600 \mathrm{MB}$ memory) at a Linux cluster machine by applying energyindependent model and energy-dependent models, respectively. However, for BEEML-PBM, the same computer could not complete the prediction of one PBEM (i.e. using the same input data) including dinucleotide interaction energies after running for almost 14 days with $\sim 12 \mathrm{~GB}$ memory. Taken together, the new program BayesPI 2 is an efficient and robust tool to analyze large data sets such as PBM.

Subsequently, PBEMs of five mouse TFs obtained by BayesPI2 based on in vitro data were used to predict TF occupancy data in the corresponding in vivo ChIP-seq data [1]. Among the five mouse TFs, three (i.e. TF_31 Zfx; TF_44 Gata4; TF_23 Tbx20) were classified as goodquality PBMs (Table 1) and the other two (i.e. TF_25 Tbx5; TF_40 Esrrb) were defined as poor PBMs (Additional file 1: Table S1) in this study. Results are shown in 
Table 2 (square root of R-square statistic) and Figure 6 ( $\mathrm{T}$ values of $\mathrm{t}$-statistic), where a linear regression model was used to evaluate the significance of dependence between the inferred PBEM from in vitro data and the measured TF tag counts from ChIP-seq experiment. For every in vivo data, the analysis was done at the top 500, 1000, 2000 ranked peaks (i.e. sorted by the number of tags found at the peak, in descending order), and all called peaks, respectively. In each selection of the topranked peaks, the same amount of bottom-ranked peaks was also considered by the regression analysis. The results tell that there are significant correlations between the predicted TF-binding affinities and the measured tag counts across different sizes of input peaks, by using inferred PBEMs from two TFs with good PBM data quality (i.e. TF_31 and TF_44). However, for the other two TFs (TF_25 and TF_40) having poor PBM data quality, the significance of such dependence is weak and different among various sizes of input data. Especially, both R-square statistic and T-values obtained from TFs with bad-quality PBMs are much smaller than those provided by the good-quality ones. Thus, poor PBM data quality may result in unreliable prediction of PBEM (i.e. algorithms may learn the background signals), which hinders any subsequent genomic analysis. Consequently, it leads to poor agreement between the estimated TF-binding affinities based on the PBEM and the measured TF occupancy data from the in vivo data.

\section{Discussion}

In this work, new quality-control parameters (i.e. PCA ellipse) were developed to assess the quality of PBM. Both single and paired PBM data quality can be illustrated in a scatter plot, where predefined control limit (i.e. $T_{2, n, p}^{2}$ ) by PCA quality-control ellipse gives a direct assessment of measurement attribute. For example, the lengths of the major and minor axes of the PCA ellipse represent the dynamical range of PBM signal intensities, and the overall difference between paired PBMs, respectively. For single $\mathrm{PBM}$, algorithm performance at the testing data may be

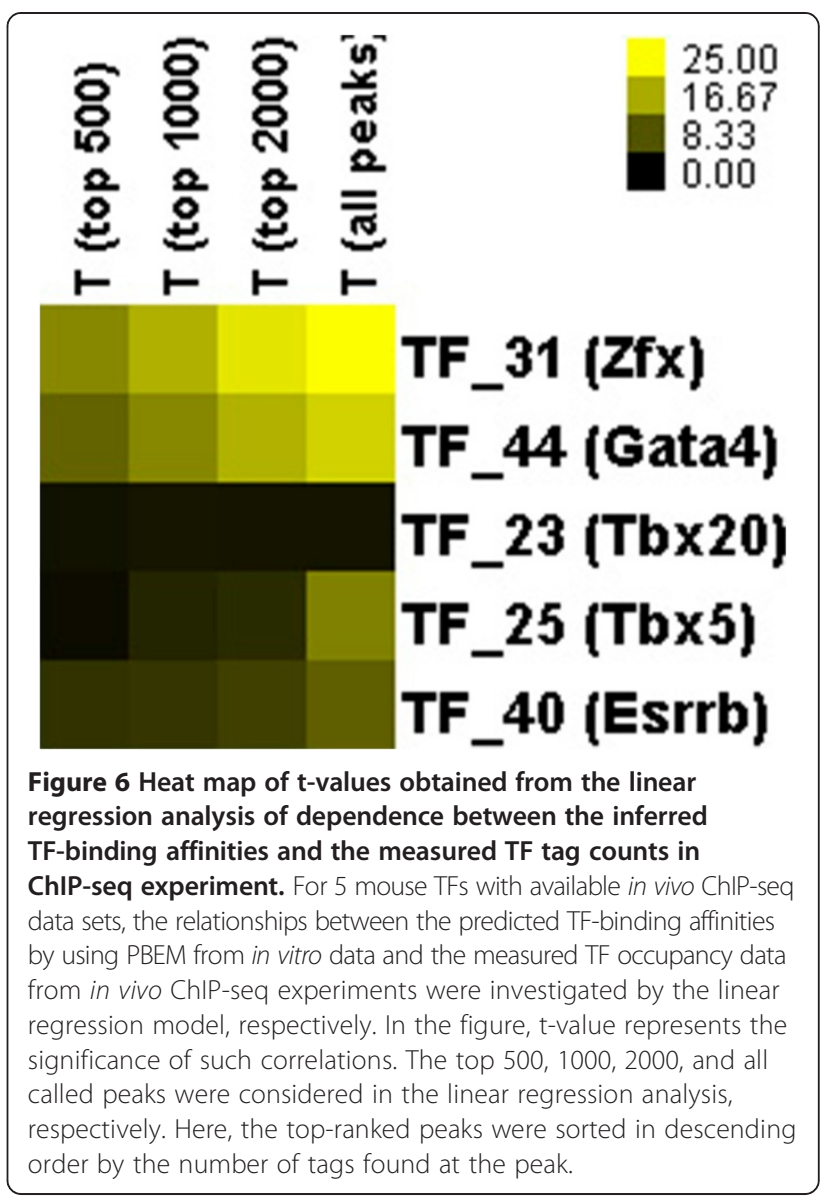

predicted for $\sim 70 \%$ of TFs based on the corresponding training data quality. For paired PBMs, a visual inspection of PCA quality-control ellipse on the scatter plot can not only identify data outliers but also tell the robustness of agreement between the two observations. On the contrary, correlation coefficient is easily affected by the data outliers (Additional file 1: Figure S7), which is not suited to measure agreement between paired observations [20]. Based on the estimated PBM quality information for 66 mouse TFs from the DREAM5 challenge, several interesting findings were revealed: for instance, both training data quality and

Table 2 Correlations between the estimated TF-binding affinities based on inferred PBEM from in vitro data and the measured TF tag counts from in vivo ChIP-seq experiment

\begin{tabular}{lrrlrrrr}
\hline ChIP-Seq & Called peaks & Rank & Paired PBMs agreement & R (top 500) & R (top 1000) & R (top 2000) & R (all peaks) \\
\hline TF_31 (Zfx) & 10338 & 8 & Good & 0.38978 & 0.35943 & 0.33566 \\
TF_44 (Gata4) & 16979 & 22 & Good & 0.29675 & 0.28458 & 0.25502 \\
TF_23 (Tbx20) & 4012 & 33 & Good & 0.077781 & 0.062428 & 0.0432945 \\
TF_25 (Tbx5) & 56352 & 41 & Bad & 0.062865 & 0.090766 & 0.073079 \\
TF_40 (Esrrb) & 21647 & 59 & Bad & 0.16334 & 0.12283 & 0.043338 \\
\hline
\end{tabular}

In the table, the first column describes ID of mouse TFs in DREAM5 challenges and the TF names to the ChIP-seq experiments; Called peaks are the number of called peaks in the ChIP-seq data; Rank represents rank order of TFs that were sorted in decreasing order of the final performance score across all tested algorithms in Figure 2 of original publication [1]; paired PBMs agreement indicates the agreement between training and testing PBMs; R is square root of R-square statistic from a linear regression model, where the relationship between the predicted TF-binding affinities and the observed ChIP-seq tag counts is investigated. 
paired PBMs agreement (Figures 2 and 3) are significantly correlated to the TF rank order according to Figure 2 of original publication [1], where 66 TFs were sorted in decreasing order by the mean of final algorithm performance scores. The results indicate that the decrease in algorithm performances across 66 mouse TFs in the DREAM5 challenge is mostly due to the gradual reduction of PBM data quality, especially the poor agreement between training and testing PBMs.

In addition to the new PBM quality-control parameters, a biophysical model of protein-DNA interactions including adjacent dinucleotide interdependence was newly implemented in C - BayesPI2, where sparse Bayesian learning approach was used to infer free-energy model parameters. The new energy-dependent model is able to recover known nucleotide interdependent effects on the binding affinities for Egr1 and Hnf4a, respectively. The results also reveal that dinucleotide interdependence often occurs at low binding energy (or information content) sites (Figure 4), which are significantly influenced by PBM data quality. Particularly, the new BayesPI2 dinucleotide energy-dependent model offers great improvement in testing prediction accuracy over the simple energy-independent model, for at least $21 \%$ of the analyzed mouse TFs (i.e. Table 1 and Additional file 1: Table S1). The new improvement might have resulted from more motif lengths searched by this study. Alternatively, the over-fitting data problem, which hampers regression-based free-energy model $[1,19]$ to estimate a large number of unknown model parameters (i.e. BEEMLPBM in R), is minimized by the Bayesian implementation of nonlinear parameter fitting.

Equipped with both the new quality-control parameters for paired PBMs and the new free-energy model including dinucleotide interdependence, 66 mouse TFs from the DREAM5 challenge were first classified into two groups (i.e. good-quality and bad-quality PBMs in Table 1 and Additional file 1: Table S1, respectively) based on the agreement between training and testing PBMs, then the algorithm accuracy on the test sets and the improvement by the energy-dependent model over the simple energyindependent model were compared between the two groups. Four new observations were revealed by this work: 1) the algorithm testing accuracy at good-quality PBMs is significantly better $\left(\mathrm{P}>7 \times 10^{-12}\right)$ than that at the badquality ones (Additional file 1: Tables S2 and S3); 2) the poor PBM data quality is protein domain specific because almost half of the bad-quality PBMs (Additional file 1: Table S1) belong to $\mathrm{C}_{2} \mathrm{H}_{2}$ DNA-binding domain (Zinc finger protein); 3) the improvement in algorithm testing prediction accuracy by using the energy-dependent model over that by the simple energy-independent model is not only associated with the PBM data quality, but also linked to the specific protein domains (i.e. Forkhead, Pou + Homeo, and bZIP); 4) the predicted binding energy-level (or information content) of a motif is significantly correlated to the quality of paired PBMs (i.e. the better paired the PBM data quality, the higher the predicted binding energy-level; Figure 5).

From these four new discoveries, two (i.e. the better the PBM quality, the better the prediction accuracy; and the better the PBM quality, the higher the binding energy-level of a motif) were observed in both the BayesPI2 predictions and the original results from the DREAM5 challenge [1]. Particularly, the predicted PBEMs from the good-quality PBMs (i.e. TF_31 and TF_44) performed significantly better than those inferred by the poor PBMs (i.e. TF_25 and TF_40), on subsequent genomic analysis in in vivo data such as ChIP-seq. Nevertheless, there is a poor correlation between the inferred TF-binding affinities and measured ChIP-seq signals for TF_23 (TBX20), a putative goodquality PBM in Table 2 and Figure 6. This may be caused by the limitation of in vivo experiment. For example, ChIPseq may not necessarily identify the direct TF-DNA interactions [24]), and TBX20 is known to directly interact with a number of proteins in regulation of gene expression [25]. The remaining two findings are supported by the literature evidences: 1) for protein domain specific PBM data quality, it is known [21] that binding of $\mathrm{C}_{2} \mathrm{H}_{2}$ zinc finger proteins are often not well measured in PBM experiments because many $\mathrm{C}_{2} \mathrm{H}_{2}$ proteins do not bind specific DNA sequences in PBM experiments; 2) for protein domain specific dinucleotide interdependence, two of the protein domains (i.e. Pou + Homeo and bZIP) were known to contain nucleotide dependence at the binding sites [21] (i.e. the homeodomain recognition helix is associated to base pair interdependency to DNA-binding, and many bZIP factors frequently bind to two distinct half-sites that may result in dinucleotide interaction). Therefore, both PBM data quality and computational modeling of protein-DNA interactions are influenced by the specific protein domains, and certain protein domains may require a free-energy model including dinucleotide interdependence to obtain precise binding energy matrix. It is important to note that the associations of both the PBM data quality and the dinucleotide energy-dependence with the protein structure classes are only revealed by this study, after applying the new PBM quality-control parameters and BayesPI2.

To minimize the effect of PBM data quality on downstream data analysis, DNA microarray experiment design from the previous works [26] might be introduced. Especially, triplet PBM experiments may be better than paired PBMs design to distinguish the experimental failure (i.e. a poor PBM data quality) from the biological failure (i.e. the TF does not bind to a DNA sequence). An alternative computational solution, to the PBM data quality issue, is to integrate k-mer sequence specific model plus feature selections into the PWM energy model (i.e. the top- 
ranked algorithm - FeatureREDUCE in the original study [1]). That is because $\mathrm{k}$-mer sequence specific model (i.e. Teams K, B, I, and Seed-and-Wobble; uses the highest-affinity $\mathrm{k}$-mer) does not consider the intensity values of PBMs when learning the motif. It is robust against the data noise that may provide a good initial seed motif for regression-based free-energy model to estimate a precise PBEM.

\section{Conclusion}

In conclusion, both the new PBM quality-control parameters and the new biophysical modeling of TF-DNA interactions including dinucleotide interdependence are developed during this work. By applying both methods on paired PBMs for 66 mouse TFs from the DREAM5 challenge, we found that: Bayesian method is robust against the data noise, and mononucleotide PWM methods do not perform similarly to more advanced dinucleotide PWM algorithms for modeling TF sequence specificity. For instance, the BayesPI2 energy-dependent model offers great improvement, for $\sim 21 \%$ of the examined TFs, in the testing prediction accuracy over that by the simple energyindependent model. Especially, the PBM data quality not only impacts the algorithm performance, but also influences the inferred binding energy-level of a motif (e.g. the better the PBM data quality, the higher the inferred binding energy-level (information content)). This work may help tremendously for future research in developing computational methods and designing PBM experiments.

\section{Methods}

\section{Principal component analysis - quality control ellipse}

To check the agreement of two measurements by principal component analysis (PCA) [27], sample mean, sample variance, and the covariance between two observations are needed. Let $X_{1}$ and $X_{2}$ be two observations (i.e. the normalized 8-mer median intensities of a mouse TF) under experiments one (i.e. training $\mathrm{PBM}$ ) and two (i.e. testing PBM), respectively. $X=\left[X_{1}, X_{2}\right]$ are two $\mathrm{n} \times 1$ vectors where $n$ is the number of observations in the experiments. The vectors of sample mean are $\bar{X}=\left[\bar{X}_{1}, \bar{X}_{2}\right]$ and the sample covariance matrix is $S=\left[\begin{array}{ll}S_{11} & S_{12} \\ S_{21} & S_{22}\end{array}\right]$. By performing PCA on the covariance matrix S, principal component coefficients are obtained: for example, $U S U=L$ where $U$ and $L$ are the eigenvectors (characteristic vectors; $U=\left[\begin{array}{ll}U_{11} & U_{12} \\ U_{21} & U_{22}\end{array}\right]$ ) and eigenvalues (characteristic roots; $L=\left[\begin{array}{cc}L_{1} & 0 \\ 0 & L_{2}\end{array}\right]$ ) of $\mathrm{S}$, respectively. Then, the $n$ variables $X$ (i.e. the vector of $X_{1}$ and $X_{2}$ ) are transformed to $n$ uncorrelated principal components $Z$ based on equation $Z=U^{\prime}[X-\bar{X}]$. Subsequently, $Z$ is scaled to $y$-score, $Y=\frac{Z}{\sqrt{L}}$ with unit variance. Finally, T-score to a pair of observations can be computed by $T^{2}=\operatorname{Diag}\left(Y^{\prime} Y\right)$ where Diag is the main diagonal of $Y Y$. It is an overall measurement of the conformance of an observation to its mean. Thus, the quantity $T^{2}$ gives a direct assessment of similarity between two measurements. Any observation vectors that produce values of $T^{2}$ greater than a predefined threshold $T_{2, n, p}^{2}$ will be out of control in a quality control (or PCA) ellipse. To compute the quality-control limit $T_{2, n, p}^{2}$, a probability value $p$ needs to be defined. For example, in this work, the limit of quality-control ellipse is three times the standard deviation $(\sim 99.73 \%)$ from sample mean, then the probability value $p$ is $\sim 0.0027$ and the $T_{2, n, p}^{2}$ is computed from $\mathrm{F}$ distribution [28]. To construct a unique quality-control ellipse in the two-dimensional case, the length of the major and minor axes, their orientation, and their interaction are needed. These information can be easily obtained from the length of the semi-major $\left(\sqrt{L_{1} T_{2, n, p}^{2}}\right)$ and semi-minor $\left(\sqrt{L_{2} T_{2, n, p}^{2}}\right)$ axes, the slope of the major $\left(V_{21} /\right.$ $\left.V_{11}\right)$ and minor $\left(-V_{11} / V_{21}\right)$ axes where $V=U L^{1 / 2}$, and the major and minor axes of the ellipse intersect at the sample mean $\bar{X}$.

\section{Quality control parameters for PBM}

Two types of quality-control parameters are defined for PBM: one is single PBM data quality and the other is paired PBM data quality. For the former, correlation coefficient between normalized signal intensities and normalized background intensities, the length of the major and minor axes of PCA ellipse, and regression coefficient are considered. Both PCA ellipse and regression coefficient were based on an $\mathrm{M}$ versus A scatter plot, where $M=\frac{1}{2}$ $\log 2($ Signal $*$ Background $), A=\log 2\left({ }^{\text {signal }} /\right.$ Background $)$. For the second one, it includes the length of the major and minor axes of PCA ellipse, correlation coefficient of normalized 8-mer median intensities between a pair of training and testing $\mathrm{PBM}$ experiments, and regression coefficient. Here, both PCA ellipse and regression coefficient are obtained from a scatter plot of normalized 8mer median intensities between a pair of PBMs. In the scatter plot, the orientation and the length of the major PCA ellipse axis is related to the first principal component, which represents the dynamical range of PBM signal intensities (i.e. with $99.73 \%$ limit of PCA quality control ellipse, $\sim 99.73 \%$ of measured signals are included in the quality ellipse); the orientation and the length of the minor PCA ellipse axis is the second principal component, which shows disagreements between the two observations. Generally, if there is good agreement between two PBMs, then a narrow PCA ellipse is obtained [4], which means that both PBMs are of good 
quality. If the PCA ellipse is wide, then the observations of the two PBMs are in poor agreement, which indicates that data quality of one of the PBMs is in question. Subsequently, the algorithm testing prediction accuracy may not be reliable due to poor agreement between training and testing PBM experiments. Taken together, the length of the major axis of the PCA ellipse (i.e. the dynamical range of $\mathrm{PBM}$ signal intensities) is an essential qualitycontrol parameter for both signal and paired PBMs.

\section{Biophysical model of protein-DNA interaction including dinucleotide interdependence}

A biophysical free-energy model of TF-DNA interactions $[5,12]$ is adopted in this work, where a Fermi-Dirac form of TF-binding probability $P(S)$ is used to estimate TFbinding energy $E(S)$ to a short stretch of DNA sequence $S$. For a detailed description of the biophysical model of protein-DNA binding, please refer to previous papers $[12,29]$. Here, the binding probability is defined by

$$
P(S)=\frac{1}{\exp (E(S)-\mu)+1}
$$

where $E(S)$ is the TF-binding free energy, and $\mu$ is the chemical potential set by the TF concentration. Please note that negative binding energy is often interpreted as the information content used by information-based weight matrix [30]. In the new BayesPI2 program, sequence-specific interaction is included in the TFbinding energy

$$
E(S)=\sum_{i=1}^{L} \sum_{a=1}^{4} E_{i, a} S_{i, a}+\sum_{i, j=1}^{L} \sum_{a, b=1}^{4} J_{i, j}^{a, b} S_{i, a} S_{j, b}
$$

where $E_{i, a}$ is the interaction energy with nucleotide $a \in(A, C, G, T)$ at position $i=1,2, \ldots L$ of the DNA sequence; $S_{i, a}$ characterizes the sequence, $S_{i, a}=1$ if $i$-th bases is $a$, otherwise $S_{i, a}=0 ; J_{i, j}^{a, b}$ is a pair of dependent energy correction (i.e. $a$ at position $i, b$ at position $j, a$, $b \in(A, C, G, T))$. In the calculation, only adjacent nucleotide interactions are considered in $J_{i, j}^{a, b}$, which reduces the number of pair-dependent energy corrections from $16 L^{2}$ to $16(L-1), L$ is the length of a motif. This simplified version of TF-DNA interaction including dinucleotide interdependence was used by an earlier paper [22] too.

\section{Sparse Bayesian learning of model parameters}

Based on the previous papers [12,29], a new Bayesian neural network framework to infer free-energy model parameters is developed. For example, to minimize errors between predicted TF-binding affinity and measured TFbinding signals, the objective function is

$$
M(w)=\beta \cdot E_{D}(D \mid w, \Lambda, \Gamma)+\alpha \cdot E_{w}(w \mid \Lambda, \Gamma)
$$

where $E_{D}=\frac{1}{2} \sum_{i=1}^{n}\left(T_{i}-Y_{i}\right)^{2}$ and $E_{w}=\frac{1}{2} \sum_{i=1}^{k} w_{k}^{2}$ are the model error function and model regularization function, respectively; $T_{i}$ is the $i$ th measured TF-binding signal and $Y_{i}$ is the predicted TF occupancy according to the proteinbinding probability $P(S) ; \alpha$ and $\beta$ are the two unknown hyperparameters that control the model parameters and the data noise level, which are determined by the input data; and $w, \Lambda, \Gamma$ represent the model parameters, the input data, and the hypothesis models (i.e. the protein-binding probability and the regularization function), respectively. After adding dinucleotide interdependence term into the protein-binding probability $P(S)$, far more model parameters need to be trained by the input data than that of an independent free-energy model [12]. To avoid over-fitting problem that may be caused by a large number of unknown model parameters, an approach similar to Bayesian sparse learning and the relevance vector machine [19] is used. For instance, the term $\alpha \cdot E_{w}$ is divided into several distinct groups

$$
\begin{aligned}
\alpha \cdot E_{w}= & \frac{1}{2}\left[\alpha_{1} w_{1}^{2}+\alpha_{2} b_{1}^{2}+\alpha_{3} \mu^{2}+\alpha_{4} \sum_{i=1}^{L} \sum_{a=1}^{4} E_{i, a}\right] \\
& +\frac{1}{2} \sum_{i, j=1 ; j-i=1}^{L} \alpha_{i, j} \sum_{a, b=1}^{4}\left(J_{i, j}^{a, b}\right)^{2}
\end{aligned}
$$

where $w_{1}$ and $b_{1}$ are the output layer parameters of neural networks, and $\mu, E_{i, a}$, and $J_{i, j}^{a, b}$ represent the chemical potential, the TF-binding energy matrix and the dinucleotide-dependent energy correction, respectively. If $\alpha_{i}$ is large, then the model parameters are close to zero, which are not important for the minimization function. Conversely, if the $\alpha_{i}$ is small, then the corresponding model parameters are important for the data fitting. In this way, sparse Bayesian learning of model parameters can be realized. Following the new Bayesian parameter minimization framework, a set of new update functions (i.e. back-propagation neural networks, Bayesian evidence approximation with R-propagation algorithm) [12] were derived and implemented in BayesPI2, a new $C$ program for inferring protein-DNA binding energy matrix.

\section{Unsupervised classification of 66 mouse TFs by PBM quality-control parameters}

An in-house made fuzzy neural gas algorithm, a combination of fuzzy logic and neural gas algorithm, was used to classify 66 TFs into two classes based on PBM qualitycontrol parameters. The neural gas algorithm uses a similar "soft-max" adaptation rule as maximum-entropy clustering and self-organizing maps (SOMs) to summarize high- 
dimensional input space (i.e. TFs) to low-dimensional reference vector space (i.e. class prototype). Then, the TF is assigned to the nearest class prototype and the fuzzy membership estimates the confidence level of the classification. Though many other machine-learning methods can be utilized to perform the same task, the Fuzzy neural gas algorithm is capable of performing unsupervised learning and capturing nonlinear relationships between the features (i.e. quality-control parameters) and sample classes [31].

\section{PBM experiments}

PBM of mouse TFs Egr1 and Hnf4a were downloaded from UniProbe database [32]. For both TFs, one of the replicate PBM experiments was used as training set and the other was used as test set to verify the newly developed BayesPI2 including dinucleotide interdependence. Both training and testing PBM experiments of 66 mouse TFs were obtained from the DREAM5 challenge [1], where normalized raw probe intensities were used to evaluate algorithm testing performance and single PBM data quality, and normalized 8-mer median intensities were used to evaluate paired PBM data quality. For all calculations, the normalization of PBM data is based on Z-score transformation of log-normalized signal intensities.

\section{ChIP-seq data}

To evaluate the predicated PBEM on in vivo data, ChIPseq data sets of five mouse TFs were obtained: Esrrb (TF_40; GEO accession GSM288355), Zfx (TF_31; GEO accession GSM288352), Tbx20 (TF_23; GEO accession GSM734426), Tbx5 (TF_25; GEO accession GSM558908), and Gata4 (TF_44; GEO accession GSM558904). For each in vivo data set, the predicted PBEM by BayesPI2 from in vitro PBM experiment was used to scan DNA sequences of all called peaks, where the TF-binding affinities were computed based on the middle 200 bases of each peak. The affinity-based analysis of DNA sequences is similar to MatrixREDUCE [33] but Fermi-Dirac form of TF-binding probability is used by BayesPI2. Then, a linear regression model is applied to test the dependence between the estimated TF-binding affinity by using in vitro PBEM and the observed TF tag counts from in vivo data. For example, the ChIP-seq tag counts represent response variables and the estimated TF-binding affinities are explanatory variables in a linear regression model. Finally, the $\mathrm{T}$ values and the correlation coefficients from the linear regression analysis are used to evaluate the significance of correlations between the estimated TF-binding affinities and the actual TF occupancy data.

\section{Computer programs}

Inferred PBEMs from PBM experiments for 66 mouse TFs by using BayesPI2, $\mathrm{C}$ version of BayesPI2 program, and
MATLAB program of PCA quality control ellipse are publically available http://folk.uio.no/junbaiw/CBayesPI2

\section{Additional files}

\section{Additional file 1: The file contains supplementary results, figures,} tables, and a description of data and code etc.

Additional file 2: The file contains figures of predicted binding energy matrices of 66 mouse TFs from DREAM5 challenge by using BayesPI2 energy-independent model. The order of figures are the same as Additional file 1: Figure S4. of original publication [1].

\section{Competing interests}

The authors declare that they have no competing interests.

\section{Authors' contributions}

JBW conceived and designed the study, implemented program in BayesPI2, performed data analysis and results interpretation, and drafted manuscript.

\section{Acknowledgments}

JBW is supported by Oslo University Hospital, Norwegian Cancer Society (DNK 2192630-2012-33376 and DNK 2192630-2013) and Norwegian Research Council NOTUR project (nn4605k). Thank Kirill Batmanov for critical reading of the manuscript.

Received: 15 April 2014 Accepted: 18 August 2014

Published: 27 August 2014

\section{References}

1. Weirauch MT, Cote A, Norel R, Annala M, Zhao Y, Riley TR, Saez-Rodriguez J, Cokelaer T, Vedenko A, Talukder S, Bussemaker HJ, Morris QD, Bulyk ML, Stolovitzky G, Hughes TR: Evaluation of methods for modeling transcription factor sequence specificity. Nat Biotechnol 2013, 31(2):126-134.

2. Yang YH, Dudoit S, Luu P, Lin DM, Peng V, Ngai J, Speed TP: Normalization for CDNA microarray data: a robust composite method addressing single and multiple slide systematic variation. Nucleic Acids Res 2002, 30(4):e15.

3. Wang J: Computational biology of genome expression and regulation-a review of microarray bioinformatics. J Environ Pathol Toxicol Oncol 2008, 27(3):157-179.

4. Wang J, Nygaard V, Smith-Sorensen B, Hovig E, Myklebost O: MArray: analysing single, replicated or reversed microarray experiments. Bioinformatics 2002, 18(8):1139-1140.

5. Djordjevic M, Sengupta AM, Shraiman BI: A biophysical approach to transcription factor binding site discovery. Genome Res 2003, 13(11):2381-2390.

6. Gerland U, Moroz JD, Hwa T: Physical constraints and functional characteristics of transcription factor-DNA interaction. Proc Natl Acad Sci U S A 2002, 99(19):12015-12020.

7. Berg OG, von Hippel PH: Selection of DNA binding sites by regulatory proteins. Statistical-mechanical theory and application to operators and promoters. J Mol Biol 1987, 193(4):723-750.

8. Granek JA, Clarke ND: Explicit equilibrium modeling of transcription-factor binding and gene regulation. Genome Biol 2005, 6(10):R87.

9. Kinney JB, Murugan A, Callan CG Jr, Cox EC: Using deep sequencing to characterize the biophysical mechanism of a transcriptional regulatory sequence. Proc Natl Acad Sci U S A 2010, 107(20):9158-9163.

10. Roider HG, Kanhere A, Manke T, Vingron M: Predicting transcription factor affinities to DNA from a biophysical model. Bioinformatics 2007, 23(2):134-141.

11. Foat BC, Morozov AV, Bussemaker HJ: Statistical mechanical modeling of genome-wide transcription factor occupancy data by MatrixREDUCE. Bioinformatics 2006, 22(14):e141-e149.

12. Wang J, Morigen: BayesPI - a new model to study protein-DNA interactions: a case study of condition-specific protein binding parameters for Yeast transcription factors. BMC Bioinformatics 2009, 10:345.

13. Zhao $Y$, Granas $D$, Stormo GD: Inferring binding energies from selected binding sites. PLOS Comput Biol 2009, 5(12):e1000590.

14. Sun W, Hu X, Lim MH, Ng CK, Choo SH, Castro DS, Drechsel D, Guillemot F, Kolatkar PR, Jauch R, Prabhakar S: TherMos: Estimating protein-DNA 
binding energies from in vivo binding profiles. Nucleic Acids Res 2013, 41(11):5555-5568.

15. Tomovic A, Oakeley EJ: Position dependencies in transcription factor binding sites. Bioinformatics 2007, 23(8):933-941.

16. Benos PV, Bulyk ML, Stormo GD: Additivity in protein-DNA interactions: how good an approximation is it? Nucleic Acids Res 2002, 30(20):4442-4451.

17. Bulyk ML, Johnson PL, Church GM: Nucleotides of transcription factor binding sites exert interdependent effects on the binding affinities of transcription factors. Nucleic Acids Res 2002, 30(5):1255-1261.

18. Man TK, Stormo GD: Non-independence of Mnt repressor-operator interaction determined by a new quantitative multiple fluorescence relative affinity (QuMFRA) assay. Nucleic Acids Res 2001, 29(12):2471-2478.

19. Tipping M: Sparse Bayesian learning and the relevance vector machine. J Mach Learn Res 2001, 1:211-244.

20. Bland JM, Altman DG: Statistical methods for assessing agreement between two methods of clinical measurement. Lancet 1986, 1(8476):307-310.

21. Jolma A, Yan J, Whitington T, Toivonen J, Nitta KR, Rastas P, Morgunova E, Enge M, Taipale M, Wei G, Palin K, Vaquerizas JM, Vincentelli R, Luscombe NM, Hughes TR, Lemaire P, Ukkonen E, Kivioja T, Taipale J: DNA-binding specificities of human transcription factors. Cell 2013, 152(1-2):327-339.

22. Zhao Y, Ruan S, Pandey M, Stormo GD: Improved models for transcription factor binding site identification using nonindependent interactions. Genetics 2012, 191 (3):781-790.

23. Chen X, Hughes TR, Morris Q: RankMotif++: a motif-search algorithm that accounts for relative ranks of K-mers in binding transcription factors. Bioinformatics 2007, 23(13):i72-i79.

24. Park PJ: ChIP-seq: advantages and challenges of a maturing technology. Nat Rev Genet 2009, 10(10):669-680

25. Stennard FA, Costa MW, Elliott DA, Rankin S, Haast SJ, Lai D, McDonald LP, Niederreither K, Dolle P, Bruneau BG, Zorn AM, Harvey RP: Cardiac T-box factor Tbx20 directly interacts with Nkx2-5, GATA4, and GATA5 in regulation of gene expression in the developing heart. Dev Biol 2003, 262(2):206-224.

26. Yang $\mathrm{YH}$, Speed T: Design issues for CDNA microarray experiments. Nat Rev Genet 2002, 3(8):579-588.

27. Jackson JE: A User's Guids to Principal Components. New York: John Wiley \& Sons, Inc; 1991.

28. Jackson JE: Principal components and factor analysis: part I- principal components. J Qual Technol 1980, 12(4):201-213.

29. Wang J: The effect of prior assumptions over the weights in BayesPI with application to study protein-DNA interactions from ChIP-based high-throughput data. BMC Bioinformatics 2010, 11:412.

30. Stormo GD, Fields DS: Specificity, free energy and information content in protein-DNA interactions. Trends Biochem Sci 1998, 23(3):109-113.

31. Wang J: A new framework for identifying combinatorial regulation of transcription factors: a case study of the yeast cell cycle. J Biomed Inform 2007, 40(6):707-725.

32. Newburger DE, Bulyk ML: UniPROBE: an online database of protein binding microarray data on protein-DNA interactions. Nucleic Acids Res 2009, 37(Database issue):D77-D82.

33. Ward LD, Bussemaker HJ: Predicting functional transcription factor binding through alignment-free and affinity-based analysis of orthologous promoter sequences. Bioinformatics 2008, 24(13):i165-i171.

\section{doi:10.1186/1471-2105-15-289}

Cite this article as: Wang: Quality versus accuracy: result of a reanalysis of protein-binding microarrays from the DREAM5 challenge by using BayesPI2 including dinucleotide interdependence. BMC Bioinformatics 2014 15:289.

\section{Submit your next manuscript to BioMed Central and take full advantage of:}

- Convenient online submission

- Thorough peer review

- No space constraints or color figure charges

- Immediate publication on acceptance

- Inclusion in PubMed, CAS, Scopus and Google Scholar

- Research which is freely available for redistribution 